\title{
Topologically protected refraction of robust kink states in valley photonic crystals
}

\author{
Fei Gao ${ }^{1 \dagger}$, Haoran Xue ${ }^{1 \dagger}$, Zhaoju Yang ${ }^{1 \star}$, Kueifu Lai ${ }^{2,3}$, Yang Yu $^{2}$, Xiao Lin ${ }^{1}$, Yidong Chong ${ }^{1,4}$, \\ Gennady Shvets $^{2 \star}$ and Baile Zhang ${ }^{1,4 \star}$
}

Recently discovered ${ }^{1,2}$ valley photonic crystals (VPCs) mimic many of the unusual properties of two-dimensional (2D) gapped valleytronic materials $s^{3-9}$. Of the utmost interest to optical communications is their ability to support topologically protected chiral edge (kink) states ${ }^{3-9}$ at the internal domain wall between two VPCs with opposite valley-Chern indices. Here we experimentally demonstrate valley-polarized kink states with polarization multiplexing in VPCs, designed from a spin-compatible four-band model. When the valley pseudospin is conserved, we show that the kink states exhibit nearly perfect out-coupling efficiency into directional beams, through the intersection between the internal domain wall and the external edge separating the VPCs from ambient space. The out-coupling behaviour remains topologically protected even when we break the spin-like polarization degree of freedom (DOF), by introducing an effective spin-orbit coupling in one of the VPC domains. This also constitutes the first realization of spin-valley locking for topological valley transport.

The valley is a binary DOF occurring in 2D honeycomb lattices, which is used in 'valleytronics' ${ }^{3-9}$ as a novel way to transport information and energy, similar to how spin is used in spintronics ${ }^{10}$. Compared to spintronic topological transport, topological transport based on the valley DOF does not require a strong spin-orbit interaction $^{11}$, and can be actively controlled in various $2 \mathrm{D}$ materials (for example, bilayer graphene) by a spatially dependent electric field $^{3,9}$ that determines the sign of the Berry curvature at the $\mathrm{K}$ and $\mathrm{K}^{\prime}$ valleys of the Brillouin zone. This enables the creation of distinct valley phases separated by internal domain walls, or 'kinks', which are populated by one-dimensional topologically protected 'kink states ${ }^{3-7}$.

In the emerging field of topological photonics ${ }^{12-20}$, recent theories that introduced the valley DOF into photonic crystals ${ }^{1,2}$ suggest that topological valley transport can provide an alternative route to achieving robust propagation of confined photons. Such a photonic platform also allows the valley and polarization (spin) DOFs to be independently manipulated, or even induced to interact ${ }^{2}$, a feature still unrealized in condensed-matter topological valley transport.

In this Letter, we experimentally demonstrate a VPC (see Fig. 1) with the following unique features. First, our design is based on a four-band model that allows polarization multiplexing. Thus two pairs of kink states with transverse-electric (TE) and transverse-magnetic (TM) polarizations can be selectively excited, whereas previous studies based on the two-band model, including the recently reported topological valley transport of sound ${ }^{21,22}$, can host only one pair of kink states in a monolayer structure. Second, the kink states can out-couple, or refract, with nearperfect efficiency into ambient space, with promising applications for directional antennas ${ }^{23}$, lasers and displays based on topological modes. This form of refraction has never been demonstrated before with topological edge modes, and is fundamentally different from ordinary light refraction at the Brewster angle, which applies only to plane waves with a specific polarization. Finally, we are able to demonstrate spin-valley-locked topological transport. For a spin-valley-locked kink state, even though the spin conservation is broken, the conserved valley symmetry can still guarantee topologically protected out-coupling behaviours.

As depicted in Fig. 1a, the designed VPC is a triangular lattice whose unit cell consists of a metallic tripod suspended between two parallel metallic plates ${ }^{20}$ (see Supplementary Information for the design procedure). The bulk band diagram is shown in Fig. $1 b$. Because the tripod geometry breaks the inversion symmetry, a bandgap $(5.8 \mathrm{GHz}<f<6.2 \mathrm{GHz})$ emerges near the $\mathrm{K}\left(\mathrm{K}^{\prime}\right)$ valleys for both TE/TM polarizations. The eigenmode profiles around the $K$ valley (for the bands labelled ' 1 ' to ' 4 ' in Fig. 1b) are plotted in Fig. 1c. For TE eigenmodes, the Poynting vector rotates clockwise/anticlockwise around the tripod in the bands ' 1 ' and '4'. This shows that the photonic valley DOF corresponds to an orbital angular momentum, similar to the valley DOF in electronic systems. For TM eigenmodes, the rotation of the Poynting vector switches to anticlockwise/clockwise in the empty region among the tripods in bands '2'/' 3 '. This shows that the polarization can act as a DOF independent of the valley DOF. The band topology analysis shows the valley-Chern indices ${ }^{6,7,24,25}$ are half-integer: $C_{\mathrm{K}}=1 / 2$ and $C_{\mathrm{K}^{\prime}}=-1 / 2$ for both TE/TM polarizations (see Methods).

Now we construct a 'kink'-type domain wall between two VPCs with opposite valley-Chern indices. As shown in Fig. 2a, the domain wall is zigzag-shaped. The lower domain (the same as in Fig. 1) has valley-Chern index $C_{\mathrm{K}\left(\mathrm{K}^{\prime}\right)}= \pm 1 / 2$. The upper domain has tripods oriented in the opposite direction as in Fig. 1, and thus exhibits the opposite valley-Chern index $C_{\mathrm{K}\left(\mathrm{K}^{\prime}\right)}=\mp 1 / 2$. The difference in valley-Chern indices across the domain wall indicates that, for each polarization, there will be two topological kink states ${ }^{3-7,24-26}$ whose propagation directions are locked to the $\mathrm{K}$ and $\mathrm{K}^{\prime}$ valleys (band diagram for a straight domain wall simulated in Fig. 2b).

Horizontal (vertical) dipole antennas oriented along the $y(z)$ direction were placed at the left end of the domain wall to launch TE (TM) polarized waves. The transmitted $H_{z}(\mathrm{TE})$ and $E_{z}(\mathrm{TM})$ fields are measured. Figure $2 \mathrm{c}$, d shows the measured transmission

${ }^{1}$ Division of Physics and Applied Physics, School of Physical and Mathematical Sciences, Nanyang Technological University, Singapore 637371, Singapore. ${ }^{2}$ School of Applied and Engineering Physics, Cornell University, Ithaca, New York 14853, USA. ${ }^{3}$ Department of Physics, University of Texas at Austin, Austin, Texas 78712, USA. ${ }^{4}$ Centre for Disruptive Photonic Technologies, Nanyang Technological University, Singapore 637371, Singapore. ${ }^{\dagger}$ These authors contributed equally to this work. ${ }^{*}$-mail: zhaojuyang@ntu.edu.sg; gshvets@cornell.edu; blzhang@ntu.edu.sg 


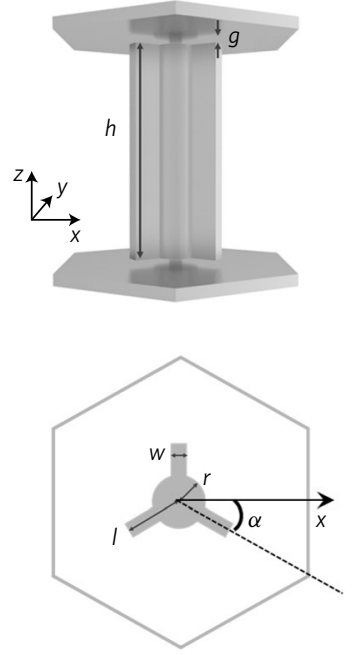

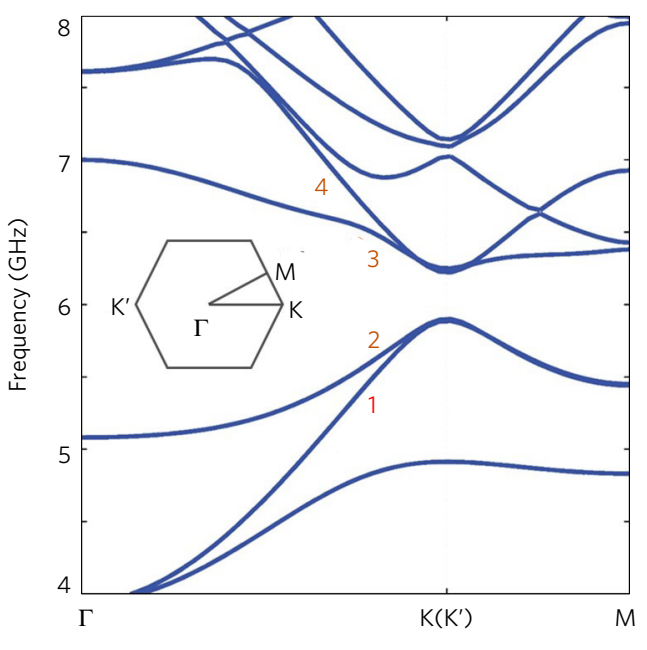

c

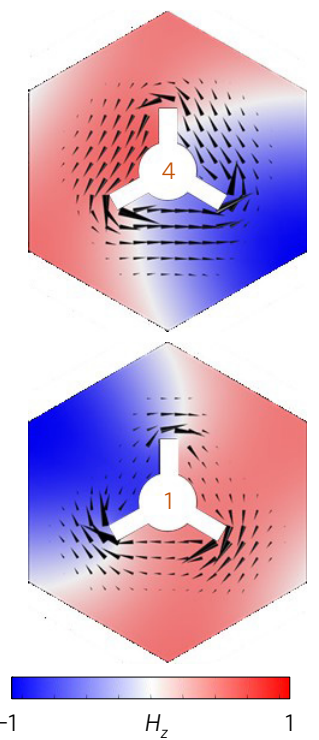

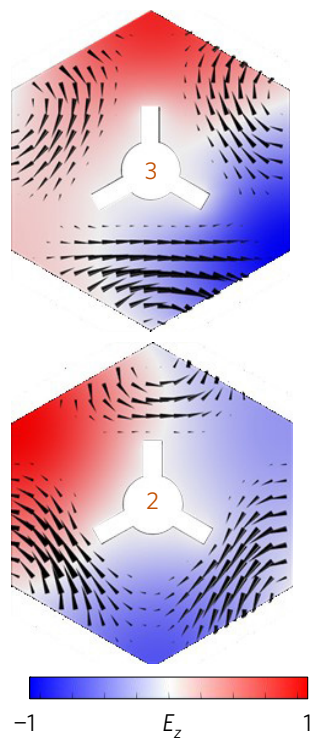

Figure 1 | Topological valley photonic crystal and its bulk band structure. $\mathbf{a}$, A unit cell of the lattice, consisting of a metallic tripod suspended in a parallel-plate waveguide. The upper and lower panels are side and top views, respectively. The tripod has height $h=34.6 \mathrm{~mm}$, inner radius $r=3.68 \mathrm{~mm}$, arm length $I=7.95 \mathrm{~mm}$, and arm width $w=2.21 \mathrm{~mm}$. Two air gaps $g=1.1 \mathrm{~mm}$ separate the tripod from the upper and lower plates (in experiment the air

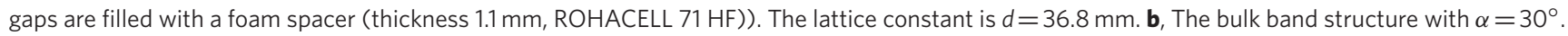
The inset shows the first Brillouin zone. Bands labelled ' 1 ' and ' 4 ' have TE polarization, while ' 2 ' and ' 3 ' have TM polarization. c, The simulated field patterns of eigenmodes in the middle $x y$ plane for corresponding bands ' 1 ' to ' 4 ', respectively. The black arrows represent Poynting power flows.

a

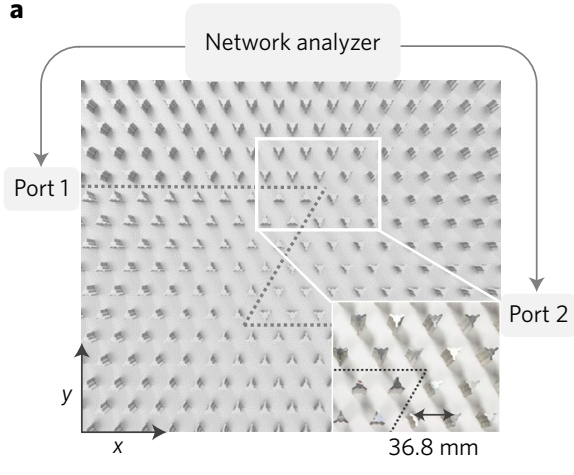

b

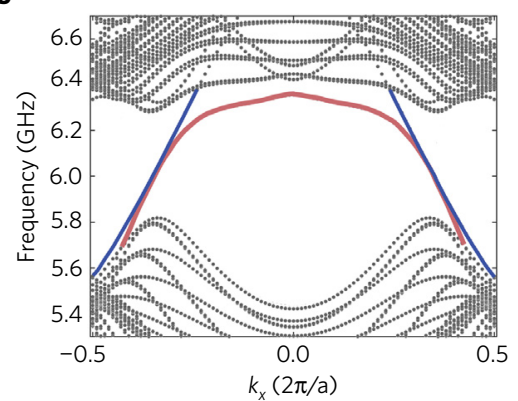

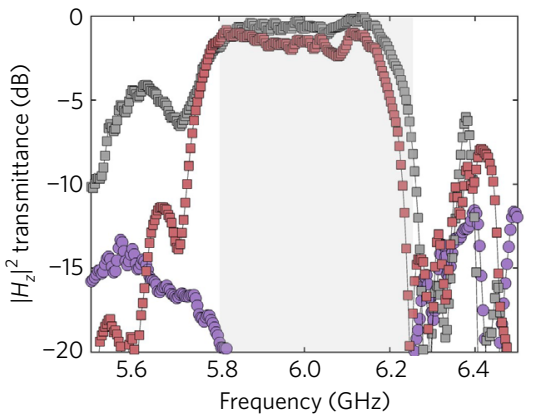

d

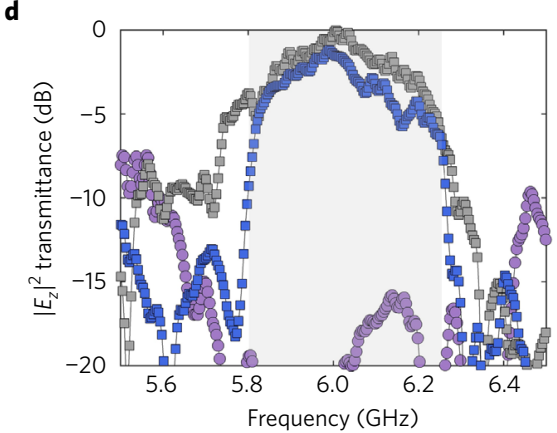

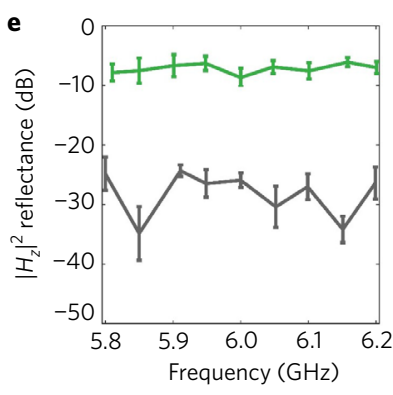

f

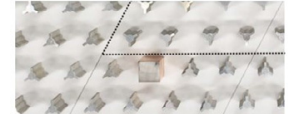

g

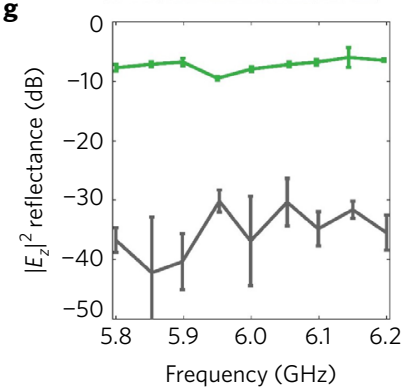

Figure $\mathbf{2}$ | Symmetry-protected topological valley kink states. a, The experimental set-up for measuring kink states. The zigzag domain wall is indicated with a dashed line. The inset is a zoomed-in photo. The upper metallic plate of the parallel-plate waveguide is removed for illustration. $\mathbf{b}$, Band diagram of kink states. The red and blue curves indicate TE and TM polarizations, respectively. c, Measured $\left|H_{z}\right|^{2}$ transmittance. Grey/red curves are for straight/zigzag domain walls, respectively. Purple curve is for the straight domain wall when the bandgap in the upper domain closes. $\mathbf{d}, M_{\text {easured }}\left|E_{z}\right|^{2}$ transmittance. Grey/blue curves are for straight/zigzag domain walls, respectively. Purple curve is for a straight domain wall when the bandgap in the upper domain closes. e,g, Measured reflectance of TE and TM modes respectively. Black curves are the reflectance from the zigzag domain wall. Green curves are for the case when a square metallic rod replaces one tripod at the domain wall, as shown in $\mathbf{f}$. Error bars represent the standard deviation of multiple measurements.

in the bandgap (5.8-6.2 GHz) along the zigzag-shaped domain wall, which is found to be comparable to the transmission along a straight domain wall of equal length (not illustrated). This demonstrates the robust transport of kink states in the absence of inter-valley scattering. When the bandgap in one domain is closed by rotating the tripods (see Supplementary Information for details), the two 

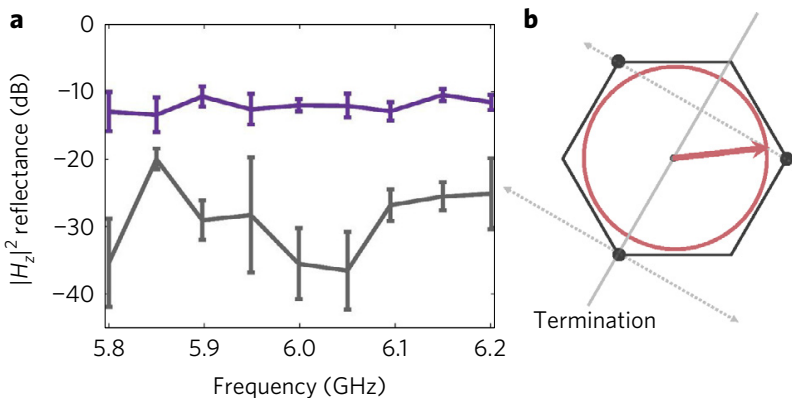

Simulation

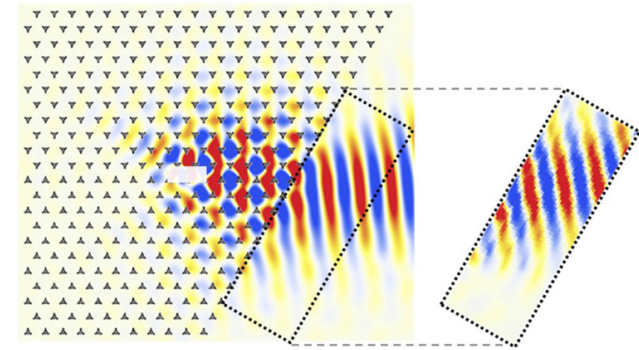

$$
-1 \longleftarrow H_{z} 1
$$

g

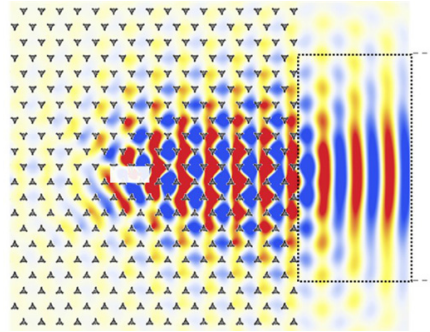

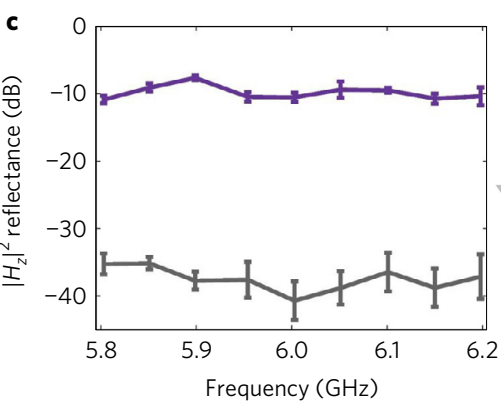

d

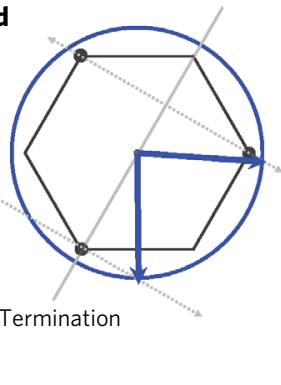

Simulation

Experiment

$\mathbf{f}$

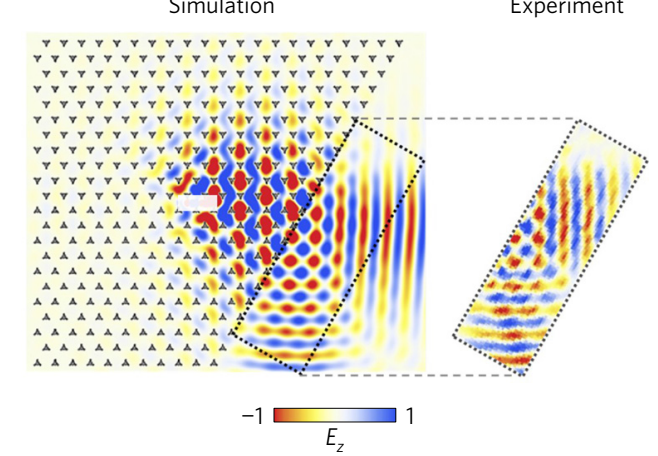

h

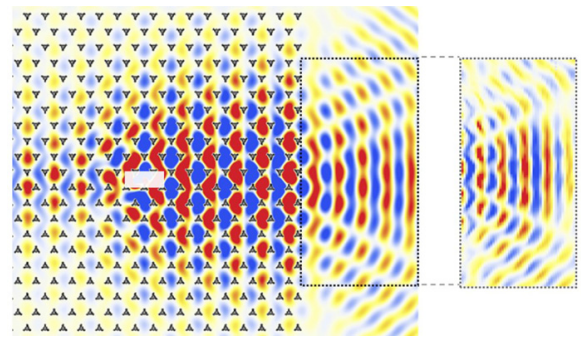

Figure 3 | Topologically protected refraction of kink states into an empty waveguide region. a, Measurement of $\left|H_{z}\right|^{2}$ reflectance for zigzag (grey) and armchair (purple) terminations. Error bars represent the standard deviation of multiple measurements. $\mathbf{b}$, The $k$-space analysis on the out-coupling of the TE polarization. The red circle represents the TE dispersion in the parallel-plate waveguide. The three black dots represent the $\mathrm{K}^{\prime}$ valley in the Brillouin zone. c, Measurement of $\left|E_{z}\right|^{2}$ reflectance for zigzag (grey) and armchair (purple) terminations. $\mathbf{d}$, The $k$-space analysis on the out-coupling of the TM polarization. The blue circle represents the TM dispersion in the parallel-plate waveguide. e,f, The refraction of TE and TM kink states through the zigzag termination, respectively. The right-hand side of each panel shows the experimentally captured field patterns. The white bars indicate the position of phase-arrayed dipoles. $\mathbf{g}, \mathbf{h}$, The refraction of TE and TM kink states through the armchair termination, respectively. The right-hand side of each panel shows the experimentally captured field patterns.

transmission bands in Fig. 2c,d, measured along the straight domain wall, drop dramatically.

We further measured the reflectance of TE/TM kink states with unidirectional excitation accomplished by an array of phased dipoles (see Supplementary Information for details). As shown in Fig. 2e,g, the reflectance from the zigzag-shaped domain wall is generally below $-25 \mathrm{~dB}$. Note that the relatively large reflectance of the TE polarization is because of the insufficient sensitivity of the magnetic probe in measuring small signals. The robustness of kink states is protected by $C_{3}$ symmetry. We break $C_{3}$ symmetry by replacing one tripod at the domain wall with a square rod (as shown in Fig. 2f). The measured reflectance in Fig. 2e,g increases by roughly two orders of magnitude.

Next, we experimentally demonstrate topologically protected refraction of the kink states at the valley-preserving zigzag termination of the VPC into the empty space in the parallel-plate waveguide (see the geometry in Fig. 3e,f). The reflectance for the TE (Fig. 3a) and TM (Fig. 3c) modes is measured with unidirectional excitation. Nearly vanishing $\left(R_{z}<3 \%\right.$, on average $\left.<0.1 \%\right)$ reflectance is observed across the entire bandgap.

Experimentally scanned empty-space field patterns at $f=6.12 \mathrm{GHz}$ are plotted in Fig. $3 \mathrm{e}\left(H_{z}\right.$ for the TE mode $)$ and
Fig. $3 \mathrm{f}$ ( $E_{z}$ for the TM mode). They reveal that the TE mode refracts into a single directional beam, whereas the TM mode refracts into two nearly orthogonal directional beams (simulation plotted in the same figures). To interpret this behaviour, we apply phase-matching conditions at the terminal interface, as shown in Fig. $3 \mathrm{~b}$ (for the TE mode) and Fig. 3d (for the TM mode). The right-moving kink states for both polarizations are locked to the $\mathrm{K}^{\prime}$ valley, as marked by three black dots $\mathbf{K}_{i}^{\prime}$ (where $i=1,2,3$ ) at the equivalent corners of Brillouin zone.

On the other hand, the waveguide dispersions are different for the two polarizations: $k_{\mathrm{TE}}=\sqrt{(\omega / c)^{2}-(\pi / d)^{2}}$ and $k_{\mathrm{TM}}=\omega / c$, as illustrated by the red/blue circles inside/outside the Brillouin zone (Fig. 3b,d). Applying the phase-matching condition to the interface parallel to $\mathbf{e}_{\text {zig }}$ requires finding the empty-space wavevectors $\mathbf{k}$ that satisfy $\mathbf{k} \cdot \mathbf{e}_{\mathrm{zig}}=\mathbf{K}_{i}^{\prime} \cdot \mathbf{e}_{\mathrm{zig}}$ and $|\mathbf{k}|=k_{\mathrm{TE}, \mathrm{TM}}$. As graphically solved in Fig. 3b,d, two solutions can be found for the TM mode but only one for the TE mode, in agreement with experiment and simulation.

For comparison, we construct an armchair termination of the VPC (Fig. 3g,h) to break the valley conservation. As shown in Fig. 3a,c, the measured reflectance increases by generally more than one order of magnitude compared to the zigzag termination. 
a

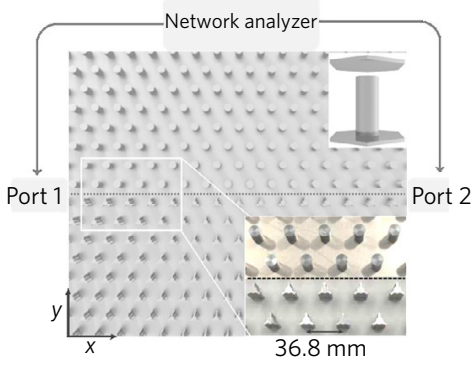

b

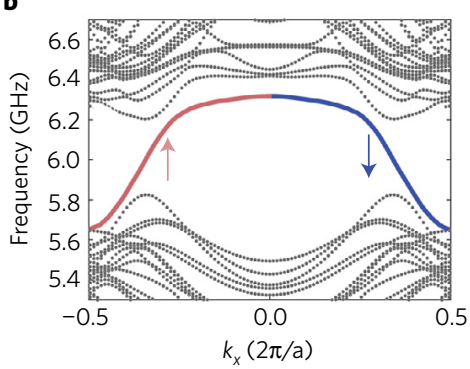

C
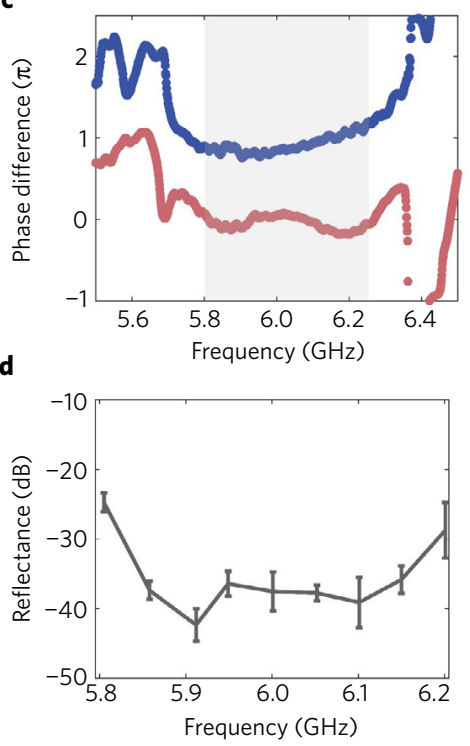

e

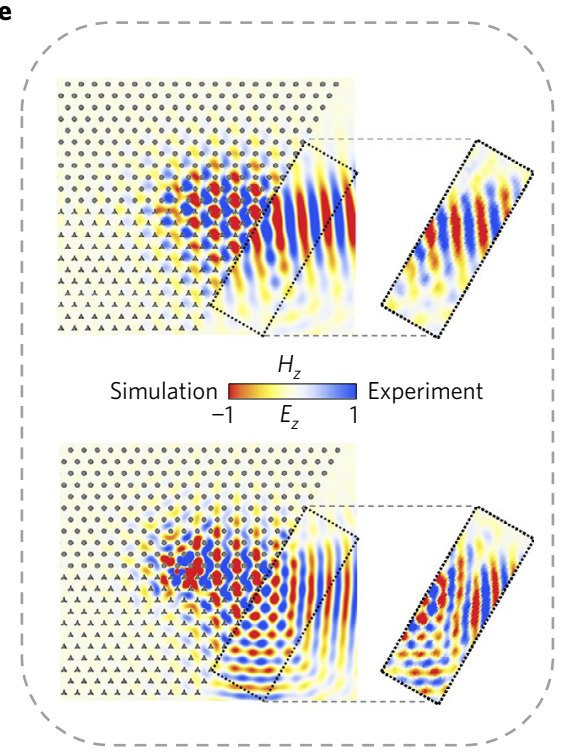

Figure 4 | Topologically protected refraction of spin-valley-locked kink states. a, The experimental set-up for measuring spin-polarized kink states. The straight domain wall is indicated with a dashed line. The upper domain is formed by metallic cylinders with radius $6.35 \mathrm{~mm}$ and height $31.3 \mathrm{~mm}$ touching the bottom plate. The upper inset shows the geometry of a metallic cylinder. The lower inset is a zoomed-in photo. The upper metallic plate of the parallel-plate waveguide is removed for illustration. b, Band diagram of kink states. The red and blue curves correspond to spin-up and spin-down kink states, respectively, as indicated by small arrows. c, Measured spins of right-moving and left-moving kink states, respectively. d, Measured reflectance of spin-up kink state from the zigzag termination. Error bars represent the standard deviation of multiple measurements. e, Out-coupling of spin-up kink state through the zigzag termination. The left/right column shows the simulated/measured field patterns, respectively. The white bars indicate the position of phase-arrayed dipoles.

To show that this anomalous refraction phenomenon at the zigzag termination arises solely from valley conservation, we study an extreme case when valley and spin DOFs are locked-that is, when the kink states are protected by both valley and spin conservation. In photonics, the in-phase and out-of-phase relations between TM $\left(E_{z}\right)$ and TE $\left(H_{z}\right)$ modes can be used to emulate the spin-up and spin-down states ${ }^{2,15-17,20}$. The spin-orbit coupling is introduced by replacing the tripods with metallic cylindrical rods touching the bottom plate ${ }^{17,20}$. As shown in Fig. 4a, a straight wall now separates the upper (spin-Hall photonic crystal) from the lower (VPC) domains. The band diagram (Fig. 4b) shows two counterpropagating spin-polarized kink states locked to the two valleys inside the bandgap (5.8-6.2 GHz). Our measurement shown in Fig. $4 c$ confirms the spin-valley locking: $E_{z}$ and $H_{z}$ fields are inphase for the right-moving kink state locked to the $\mathrm{K}^{\prime}$ valley, and out-of-phase for the left-moving kink state locked to the $\mathrm{K}$ valley.

The right-moving (spin-up) kink state was then selectively excited to test the topologically protected refraction through the zigzag termination (Fig. 4e). Negligible reflectance is measured, as shown in Fig. 4d. In the empty waveguide region, TE and TM modes are separated into different directional beams, as shown in Fig. 4e, similar to Fig. 3e,f without spin-valley locking. This shows that, even though the spin conservation is broken by the spin-coupling termination, the conserved valley symmetry can still enable 'perfect' refraction.

The above results demonstrate the topologically protected refraction of robust kink states into the ambient space defined by an unpatterned parallel-plate waveguide. The polarization multiplexing can double data capacity to support robust and high-speed wireless and optical data networks ${ }^{27,28}$. Due to the high efficiency of the coupling between the topological modes and free space modes, we anticipate many practical applications for directional antennas, lasers, and other communication devices across the electromagnetic spectrum. During preparation of this work, two related reports ${ }^{29,30}$ of experimental realizations of photonic valley edge states were brought to our attention. The fundamental difference of our work is the demonstration of topologically protected refraction.

\section{Methods}

Methods, including statements of data availability and any associated accession codes and references, are available in the online version of this paper.

Received 4 June 2017; accepted 27 September 2017;

published online 13 November 2017

\section{References}

1. Ma, T. \& Shvets, G. All-Si valley-Hall photonic topological insulator. New J. Phys. 18, 025012 (2016).

2. Dong, J. et al. Valley Photonic crystals for control of spin and topology. Nat. Mater. 16, 298-302 (2017).

3. Martin, I., Blanter, Y. M. \& Morpurgo, A. F. Topological confinement in bilayer graphene. Phys. Rev. Lett. 100, 036804 (2008).

4. Semenoff, G. W., Semenoff, V. \& Zhou, F. Domain walls in gapped graphene. Phys. Rev. Lett. 101, 087204 (2008).

5. Yao, W., Yang, S. \& Niu, Q. Edge states in graphene: from gapped flat-band to gapless chiral modes. Phys. Rev. Lett. 102, 096801 (2009).

6. Zhang, F. et al. Spontaneous quantum Hall states in chirally stacked few-layer graphene systems. Phys. Rev. Lett. 106, 156801 (2011).

7. Zhang, F., MacDonald, A. H. \& Mele, E. J. Valley Chern numbers and boundary modes in gapped bilayer graphene. Proc. Natl Acad. Sci. USA 110, 10546-10551 (2013).

8. Ju, L. et al. Topological valley transport at bilayer graphene domain walls. Nature 520, 650-655 (2015).

9. Li, J. et al. Gate-controlled topological conducting channels in bilayer graphene. Nat. Nanotech. 11, 1060-1065 (2016).

10. Zutic, I., Fabian, J. \& Sarma, S. Spintronics: fundamentals and applications. Rev. Mod. Phys. 76, 323-410 (2004).

11. Kane, C. L. \& Mele, E. J. Quantum spin Hall effect in graphene. Phys. Rev. Lett. 95, 226801 (2005).

12. Wang, Z., Chong, Y., Joannopoulos, J. D. \& Soljacic, M. Observation of unidirectional backscattering-immune topological electromagnetic states. Nature 461, 772-775 (2009). 
13. Hafezi, M., Demler, E. A., Lukin, M. D. \& Taylor, J. M. Robust optical delay lines with topological protection. Nat. Phys. 7, 907-912 (2011).

14. Rechtsman, M. C. et al. Photonic floquet topological insulators. Nature 496, 196-200 (2013).

15. Khanikaev, A. B. et al. Photonic topological insulators. Nat. Mater. 12, 233-239 (2013)

16. Chen, W. et al. Experimental realization of photonic topological insulator in a uniaxial metacrystal waveguide. Nat. Commun. 5, 5782 (2014).

17. Ma, T., Khanikaev, A. B., Mousavi, S. H. \& Shvets, G. Guiding electromagnetic waves around sharp corners: topologically protected photonic transport in metawaveguides. Phys. Rev. Lett. 114, 127401 (2015)

18. Gao, F. et al. Probing topological protection using a designer surface plasmon structure. Nat. Commun. 7, 11619 (2016).

19. Lu, L., Joannopoulos, J. D. \& Soljacic, M. Topological states in photonic systems. Nat. Phys. 12, 626-629 (2016).

20. Ma, T. \& Shvets, G. Scattering-free optical edge states between heterogeneous photonic topological insulators. Phys. Rev. B 95, 165102 (2017).

21. Lu, J., Qiu, C., Ke, M. \& Liu, Z. Valley vortex states in sonic crystals. Phys. Rev. Lett. 116, 093901 (2016).

22. Lu, J. et al. Observation of topological valley transport of sound in sonic crystals. Nat. Phys. 13, 369-374 (2017).

23. Fleury, R., Khanikaev, A. B. \& Alu, A. Floquet topological insulators for sound. Nat. Commun. 7, 11744 (2016).

24. Ezawa, M. Topological Kirchholff law and bulk-edge correspondance for valley Chern and spin-valley Chern numbers. Phys. Rev. B 88, 161406 (2013).

25. Ezawa, M. Symmetry protected topological charge in symmetry broken phase: spin-Chern, spin-valley-Chern and mirror-Chern number. Phys. Lett. A 378, 1180-1184 (2014).

26. Li, J., Morpurgo, A. F., Buttiker, M. \& Martin, I. Marginality of bulk-edge correspondence for single-valley Hamiltonians. Phys. Rev. B 82, 245404 (2010).

27. Wellbrock, G. \& Xia, T. J. The road to $100 \mathrm{G}$ deployment. IEEE Commun. Mag. 48, S14-S18 (2010).
28. Chen, Z.-Y. et al. Use of polarization freedom beyond polarization-division multiplexing to support high-speed and spectral-efficient data transmission. Light Sci. Appl. 6, e16207 (2017)

29. Wu, X. et al. Direct observation of valley-polarized topological edge states in a designer surface plasmon crystals. Preprint at http://arXiv.org/abs/1703.04570 (2017).

30. Noh, J. et al. Observation of photonic topological valley-Hall edge states. Preprint at http://arXiv.org/abs/1706.00059 (2017).

\section{Acknowledgements}

This work was sponsored by Nanyang Technological University for NAP Start-up Grants, Singapore Ministry of Education under Grants No. MOE2015-T2-1-070,

MOE2015-T2-2-008, MOE2016-T3-1-006 and Tier 1 RG174/16 (S). K.L., Y.Y. and G.S.

acknowledge the support of the Air Force Office of Scientific Research under a Grant

No. FA9550-15-1-0075, and the Army Research Office under a Grant

No. W911NF-16-1-0319.

\section{Author contributions}

All authors contributed extensively to this work. F.G., H.X. and Z.Y. fabricated structures and performed measurements. F.G., Z.Y., Y.Y. and X.L. performed simulation. F.G. and Z.Y. provided major theoretical analysis. K.L. designed part of the unidirectional excitation experiment. Y.C., G.S., and B.Z. supervised the project.

\section{Additional information}

Supplementary information is available in the online version of the paper. Reprints and permissions information is available online at www.nature.com/reprints. Publisher's note: Springer Nature remains neutral with regard to jurisdictional claims in published maps and institutional affiliations. Correspondence and requests for materials should be addressed to Z.Y., G.S. or B.Z.

\section{Competing financial interests}

The authors declare no competing financial interests. 


\section{Methods}

Fabrication and simulation. The aluminium tripods are fabricated with the wire Electric Discharge Machining (wire EDM) method. The band diagrams are simulated with the first-principles electromagnetic simulation software COMSOL Multiphysics, where the aluminium tripods used in experiments are modelled as a perfectly electric conductor (PEC). The dispersion shown in Fig. $2 \mathrm{~b}$ was performed with a supercell that contains 10 tripods on each side of the interface. The field patterns are simulated with CST Microwave Studio. For the TM mode excitation, a vertical dipole with length $34 \mathrm{~mm}$ is placed in the middle of the two-parallel-plate waveguide. For the TE mode excitation, a horizontal dipole with length $34 \mathrm{~mm}$ is placed in the middle of the two-parallel-plate waveguide.

Dirac Hamiltonian and valley-Chern number. Photonic lattices with $C_{6 v}$ symmetries are known to possess an extra discrete degree of freedom: the valley, which refers to the proximity of propagating electromagnetic waves to one of the two high-symmetry corners at $\mathrm{K}=\left(4 \pi / 3 a_{0}, 0\right)$ and $\mathrm{K}^{\prime}=\left(-4 \pi / 3 a_{0}, 0\right)$ of the Brillouin zone. Under a broad set of perturbations ${ }^{1}$ that do not scatter photons from one valley into another, the valley is conserved. Under the valley conservation assumption, it becomes appropriate to consider a restricted topological phase of photons that is defined in only one of the two valleys. Such phases are characterized by restricted (valley-projected) half-integer Chern numbers associated with their valley: $C_{\mathrm{K}}=1 / 2$ and $C_{\mathrm{K}^{\prime}}=-1 / 2$ obtained by integrating the Berry curvature over a restricted region of the Brillouin zone that coincides with one of the valleys. The bulk-boundary correspondence principle prohibits topological edge states at the interface between a valley-projected topological phase and a topologically trivial phase. However, the kink states at the domain wall between VPCs with half-integer spin-valley-Chern numbers of the opposite sign are allowed.

Formally, the band topology of the VPC can be described by a massive Dirac Hamiltonian $H=v_{\mathrm{D}}\left(\delta k_{x} \tau_{z} s_{0} \sigma_{x}+\delta k_{y} \tau_{0} s_{0} \sigma_{y}\right)+m \tau_{0} s_{0} \sigma_{z}$. Here, $v_{\mathrm{D}}$ is the group velocity, $\left(\delta k_{x}, \delta k_{y}\right)$ is the momentum deviation from $\mathrm{K}\left(\mathrm{K}^{\prime}\right)$ point, $\sigma_{x, y, z}, \tau_{z}$, are the Pauli matrices acting on orbital and valley state vectors, respectively, and $\tau_{0}, s_{0}$ are unit matrices acting on valley and polarization state vectors, respectively. $m$ is the effective mass induced by inversion-symmetry-breaking of the tripod geometry. This Hamiltonian produces a nontrivial Berry curvature $\Omega$ in the lower TE/TM bands, whose integration near the $\mathrm{K}\left(\mathrm{K}^{\prime}\right)$ valley gives rise to the valley-Chern number $C_{\mathrm{K}}=1 / 2$ and $C_{\mathrm{K}^{\prime}}=-1 / 2$ for both TE/TM polarizations. Note that the integration of Berry curvature over the whole Brillouin zone is zero because of time-reversal symmetry.

Data availability. The data that support the plots within this paper and other findings of this study are available from the corresponding author upon reasonable request. 